\title{
The optimization design of the triangular mesh of the lamella ellipsoid reticulated shell and the comparative analysis of its static performance
}

\author{
Shuai Chong ${ }^{1}$, Lili Huang ${ }^{2}$, Junchao $\mathrm{Cao}^{1}$ and Xiaoyang $\mathrm{Lu}^{1 *}$ \\ ${ }^{1}$ Institute of Engineering Mechanics, Shandong Jianzhu University, Ji Nan, Shan Dong, 250101, China. \\ ${ }^{2}$ School of Materials Science and Engineering, Shandong Jianzhu University, Ji Nan, Shan Dong, 250101, China.
}

\begin{abstract}
Using the traditional geometric principle and ANSYS software Parametric Design Language (APDL), the optimization method is given for the triangular shell mesh of lamella ellipsoid. The mechanical properties of two types of ellipsoidal reticulated shells, optimized isosceles shell (OIS) and traditional shell (TS), are analyzed by comparison with ANSYS software. The results show that the ellipsoidal reticulated shell composed of an optimized isosceles triangle mesh has better mechanical performance and is widely used in engineering.
\end{abstract}

\section{Introduction}

The type and mesh division of reticulated shell structure not only affect its architectural modeling but also affect the mechanical performance of the structure itself $[1,2]$. The research on the mesh optimization of spherical reticulated shell with the equilateral triangle element $[3,4]$ can make the reticulated shell have the characteristics of reasonable structural force, single basic rod, easy assembly and simple construction. However, there are few studies on ellipsoidal reticulated shells.

In this paper, combined with the connection characteristics of the nodes of the lamella ellipsoid reticulated shell, the optimization of the triangular grid of the lamella ellipsoid reticulated shell is carried out, which not only makes the structure stress more reasonable, but also reduces the type of rods of this kind of ellipsoid reticulated shell, which brings great convenience to the engineering construction.

\section{Geometric description of structure}

The main geometric parameters of ellipsoidal reticulated shell are as follows: semi-major axes $\mathrm{R}_{1}$, semi-minor axes $\mathrm{R}_{2}$, rise $\mathrm{f}$, Number of shares of circular symmetric region $\mathrm{Kn}$, Number of radial node turns Nx (figure 1.).

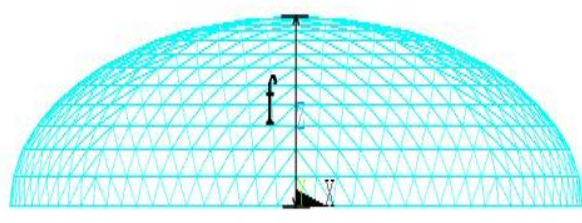

(a)

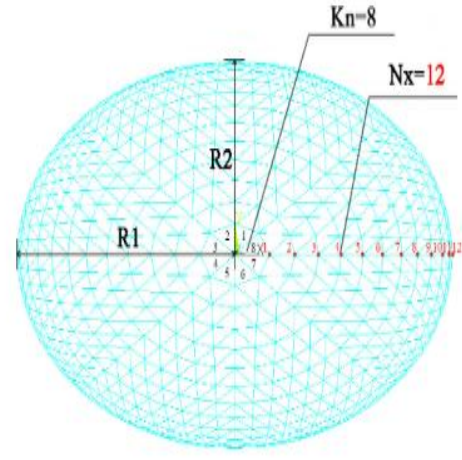

(b)

Figure 1. Schematic diagram of structural geometric parameters.

\footnotetext{
*Corresponding author’s e-mail: zhdwr@outlook.com
} 


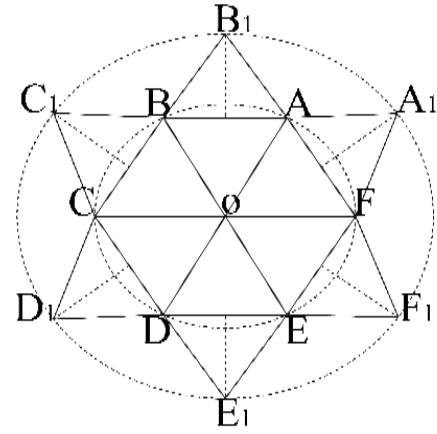

(a)

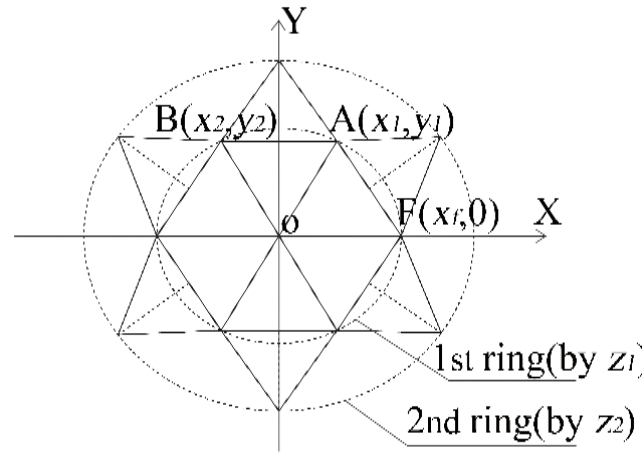

(b)

Figure 2. Schematic diagram of geometry.

\section{Optimization of isosceles triangular mesh in ellipsoid shell}

\subsection{Geometric process}

(1). To determine the positi on of the first ellipse nodes: If $\mathrm{Kn}=6$, the ellipse of the first ring is divided into 6 segments, where $\mathrm{AB}=\mathrm{BC}=\mathrm{CD}=\mathrm{DE}=\mathrm{DF}$ (figure 2.(a)). Points $\mathrm{O}$ and $\mathrm{A}-\mathrm{F}$ are nodes of the reticulated shell of the ellipsoid, points $\mathrm{A}-\mathrm{F}$ are on the ellipsoid locus equation of the first ring, point $\mathrm{O}$ is the vertex of the reticulated shell.

(2). To determine the positi on of the next ellipse nodes: Make the perpendicular bisector of the length of the last ellipse string $(\mathrm{AB}, \mathrm{BC}, \mathrm{CD}, \mathrm{DE}, \mathrm{EF})$ respectively. The next ring nodes is located on the perpendicular bisector (figure 2. (a)). The virtual line in the figure is the central perpendicular line of each loop-direction bar of the upper ring, obviously, $\triangle \mathrm{ABB}_{1}, \triangle \mathrm{BCC}_{1}, \triangle \mathrm{CDD}_{1}, \triangle \mathrm{DEE}_{1}$, $\triangle \mathrm{EFF}_{1}, \triangle \mathrm{FAA}_{1}$ are isosceles triangle, and $\triangle \mathrm{ABB}_{1} \cong \triangle \mathrm{DEE}_{1}$ $\triangle \mathrm{BCC}_{1} \cong \triangle \mathrm{CDD}_{1} \cong \triangle \mathrm{EFF}_{1} \cong \triangle \mathrm{FAA}_{1}$.

(3). Repeat the (2) process until it is connected to the bottom of the ellipsoid.

\section{2 mathematical model}

If $\mathrm{R}_{1}=a, \mathrm{R}_{2}=b, \mathrm{Kn}=c, \mathrm{Nx}=d, \mathrm{f}=e$, standard ellipsoid equations in the rectangular space coordinate:

$$
\frac{x^{2}}{a^{2}}+\frac{y^{2}}{b^{2}}+\frac{z^{2}}{e^{2}}=1
$$

$\mathrm{O}(0,0, \mathrm{e})$ is the vertex coordinates of the reticulated shell, and and the plane of the $\mathrm{i}$-th ring is $z=z_{i}$, $(i=$ $1,2,3, \ldots, d$.).

The elliptic curve equation of each ring is derived from the plane $z=z_{i}$ intercept ellipsoid[5]:

$$
f(x, y)=\left\{\begin{aligned}
\frac{x^{2}}{a^{2}}+\frac{y^{2}}{b^{2}} & =1-\frac{z_{i}^{2}}{e^{2}} \\
z & =z_{i}
\end{aligned}\right.
$$

If $\mathrm{A}\left(x_{1}, y_{1}\right), \mathrm{B}\left(x_{2}, y_{2}\right)$, the distance between $\mathrm{A}$ and $\mathrm{B}$ is:

$$
|A B|=\sqrt{\left(x_{2}-x_{1}\right)^{2}+\left(y_{2}-y_{1}\right)^{2}}
$$

main steps:

(1). Find the node coordinates of the first ring: the plane of the first ring is $z=z_{1}, z_{1}$ is determined by the proportion [6] of the rise of the ellipsoid shell. Establish plane rectangular coordinate with point $\mathrm{O}$ as the origin of coordinates (figure 2. (b)). The node coordinates of the first ring have a total of $\mathrm{Kn}=c$, and all nodes are symmetrically distributed on the elliptic curve. Take the first quadrant of the ellipse to study, get all the node coordinates of the first quadrant, then get all the node coordinates of the ring.

The starting node $\mathrm{F}$ of the first ring is located on the $\mathrm{X}$-axis (figure 2.(c)). According to equation (2), the coordinates of $\mathrm{F}$ point $\left(x_{f}, 0\right)$ can be directly obtained.

The elliptic chord length of the same ring can be obtained by the distance of two adjacent points through formula (3). Since the length of each string is the same, ( $\mathrm{m}-1)$ independent equations can be combined ( $\mathrm{m}$ is the number of nodes in the first quadrant):

$\left\{\begin{array}{c}\sqrt{\left(x_{1}-x_{f}\right)^{2}+\left(y_{1}-0\right)^{2}}=\sqrt{\left(x_{2}-x_{1}\right)^{2}+\left(y_{2}-y_{1}\right)^{2}} \\ \sqrt{\left(x_{2}-x_{1}\right)^{2}+\left(y_{2}-y_{1}\right)^{2}}=\sqrt{\left(x_{3}-x_{2}\right)^{2}+\left(y_{3}-y_{2}\right)^{2}} \\ \sqrt{\left(x_{3}-x_{2}\right)^{2}+\left(y_{3}-y_{2}\right)^{2}}=\sqrt{\left(x_{4}-x_{3}\right)^{2}+\left(y_{4}-y_{3}\right)^{2}} \\ \cdots \\ \sqrt{\left(x_{m-1}-x_{m}\right)^{2}+\left(y_{m-1}-y_{m}\right)^{2}}=2 x_{m}\end{array}\right.$

By combining equation (1) with equations (5), the coordinates of each node in the first quadrant can be obtained, and the coordinates of all nodes in the first ring can be known from the symmetry.

(2). Find the equation of the midperpendicular of the chords of the top ring: according to the node coordinates of the first ring, the coordinates of the chord midpoints and the normal vectors of the chords of the first ring are obtained.Using them to find he equation for the midperpendicular. Given the coordinates of the ends of the chords are $\left(x_{j}, y_{j}\right)$ and $\left(x_{j+1}, y_{j+1}\right), j$ represents the $j$-th chord $\left(j=1,2,3, \ldots, \mathrm{K}_{\mathrm{n}}\right)$, where the equation of the perpendicular bisector is: 


$$
g_{j}=\left\{\begin{array}{l}
x=0, y_{j}=y_{j+1} \\
y=0, x_{j}=x_{j+1} \\
\frac{x_{j}-x_{j+1}}{y_{j+1}-y_{j}}\left(x-\frac{x_{j}-x_{j+1}}{2}\right)+\frac{y_{j}+y_{j+1}}{2}, \text { else }
\end{array}\right.
$$

(3). Find the node coordinates of the next ring: the plane of the next ring is $z=z_{2}$, and the equations (5) of each perpendicular bisector obtained from 2 are respectively connected with equation (2), and the solutions are the node coordinates of the ring.

(4). The node coordinates of each ring can be obtained by repeating the above process.

\section{Shell parameter modeling}

The east-west long axis of the National Grand Theatre is $212.2 \mathrm{~m}$, and the south-north short axis is $143.64 \mathrm{~m}$. The total height is $46.285 \mathrm{~m}$. The ratio of the length axis of the National Grand Theatre is $R_{1} / R_{2}$, and the ratio of the rise to the length axis is $f / R_{1}$ for reference[7]. The selected parameter of ellipsoid reticulated shell is: $R_{1}=90 \mathrm{~m}$, $\mathrm{R}_{2}=54 \mathrm{~m}, \mathrm{f}=18 \mathrm{~m}, \mathrm{Kn}=18, \mathrm{Nx}=8$.

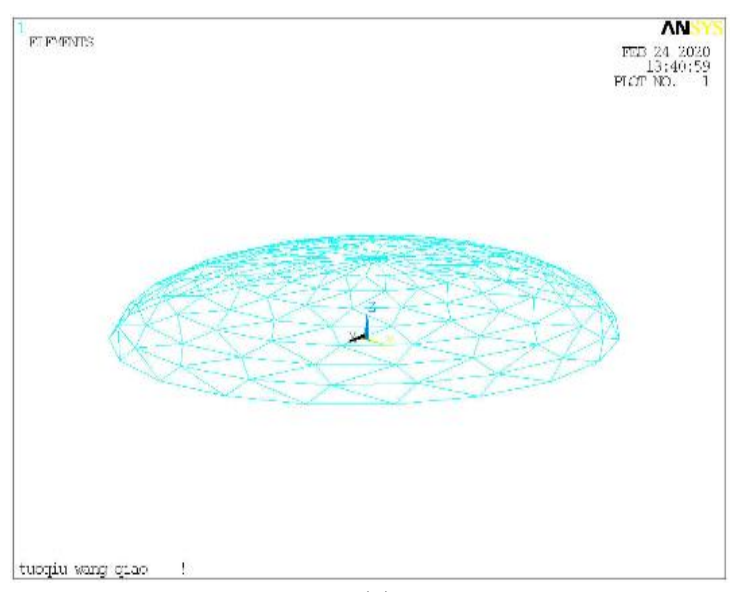

(a)
ANSYS software apdl parametric design language[8] was used for traditional form and optimization form modeling.

It can be seen from figure 3 that before grid optimization (figure 3.(a)), the lamella ellipsoid reticulated shell is composed of a variety of triangular meshes, with various lengths and types of rods. After the grid optimization (figure 3.(b)), the grid of the lamella ellipsoid reticulated shell becomes several regular congruent isosceles triangle, and the rod types are greatly reduced. In order to explore the mechanical properties of the two, it is necessary to analyze and compare the static properties.

\section{Static analysis comparison}

Static analysis of the structure, which applying the niform load of $2.35 \mathrm{kN} / \mathrm{m} 2[9]$, which the material of the structural rods is Q345 steel pipe and the section size is $\Phi 168 \times 7.0$, which considering the self-weight of the structure and the form of structural supports are fixed supports, which the allowable structure displacement is $1 / 400$ of the shortdirectional span[10]. Maximum displacement (MD), allowable displacement (AD), maximum stress (MS), allowable stress (AS), structure type (ST).

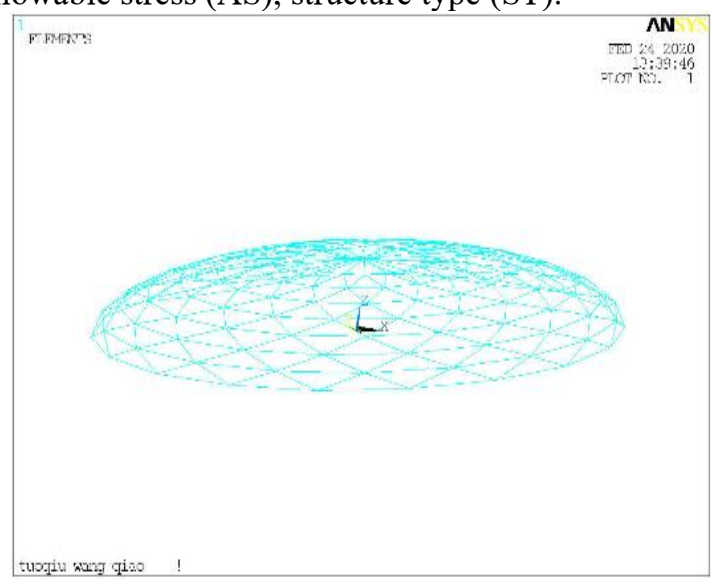

(b)

Figure 3. Comparison of models before and after optimization.

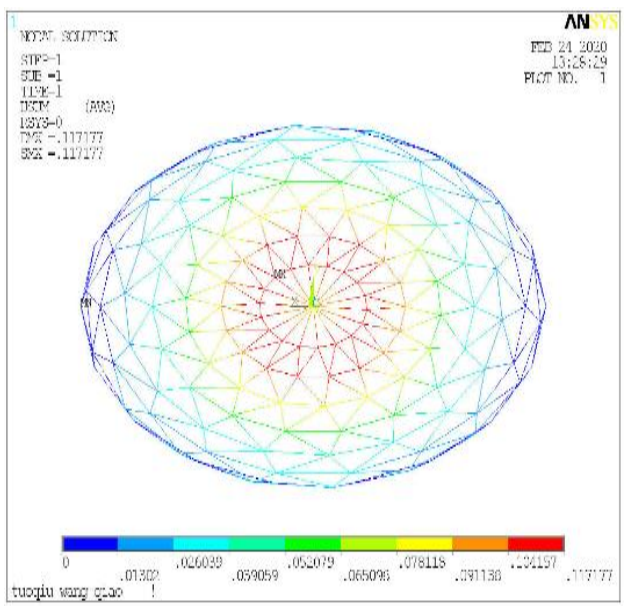

(a)

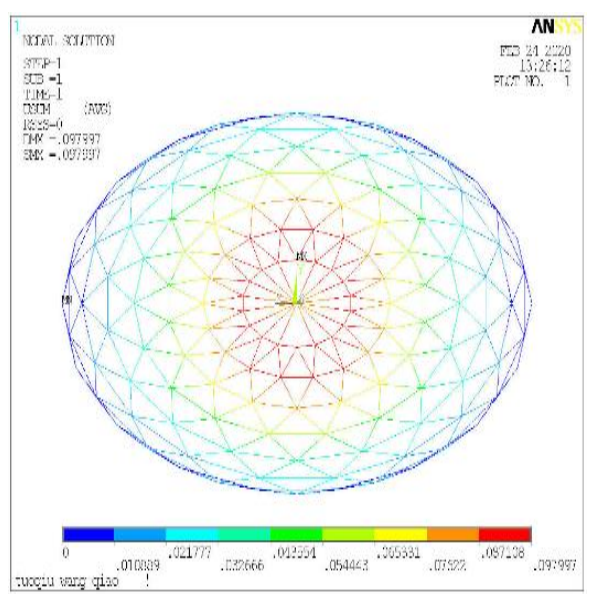

(b)

Figure 4.Comparison of maximum displacement diagram. 


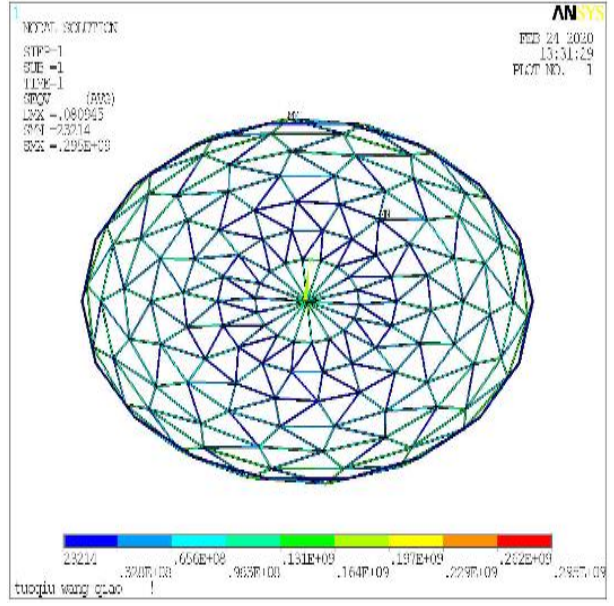

(a)

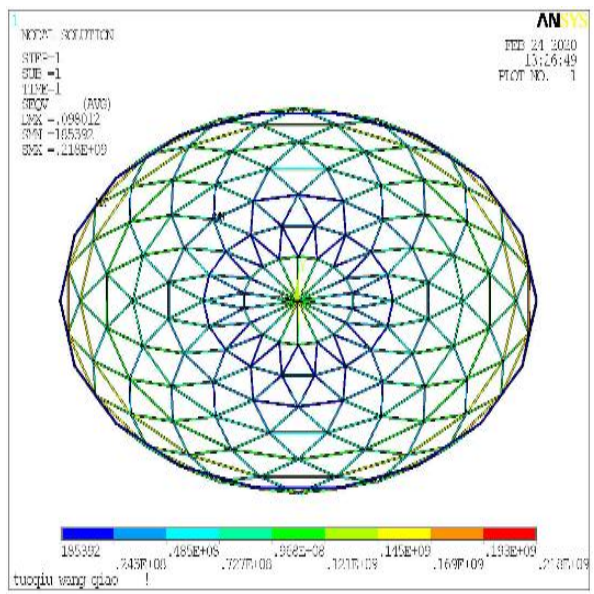

(b)

Figure 5.Comparison of maximum stress diagram.

Note: (a) represents TS, (b) represents OIS.

The results are as follows:

Table 1. Comparison of MD and MS between TS and OIS.

\begin{tabular}{ccccc}
\hline ST & MD $(\mathrm{cm})$ & AD $(\mathrm{cm})$ & MS (MPa) & AS (MPa) \\
\hline TS & 11.72 & 13.50 & 295 & 345 \\
OIS & 9.80 & 13.50 & 218 & 345 \\
\hline
\end{tabular}

The following conclusions can be drawn from figure 4-5. and table 1. that:

(1). The MD of two types of reticulated shells is concentrated in the circumferential rods and radial rods in the first few turns of the superstructure, the stiffness of the superstructure is smaller than that of the substructure, and the mesh optimization does not significantly change the position of the MD.

(2). Both types of reticulated shells meet the structural strength requirements, and the most adverse stress is compressive stress.
(3) The MD and MS of the reticulated shell after optimization are significantly reduced compared with that before optimization.

The changes of MD and MS of two types of reticulated shells, as well as the number of structural bars (NSR) and structural nodes (NSN), will be investigated when $\mathrm{Nx}=6$, 8,10 and $\mathrm{Kn}=16-20$ (step size is 2), as shown in table 2, 3 and 4.

Table 2. $\mathrm{Nx}=6$, contrasting between TS and OIS under different Kn.

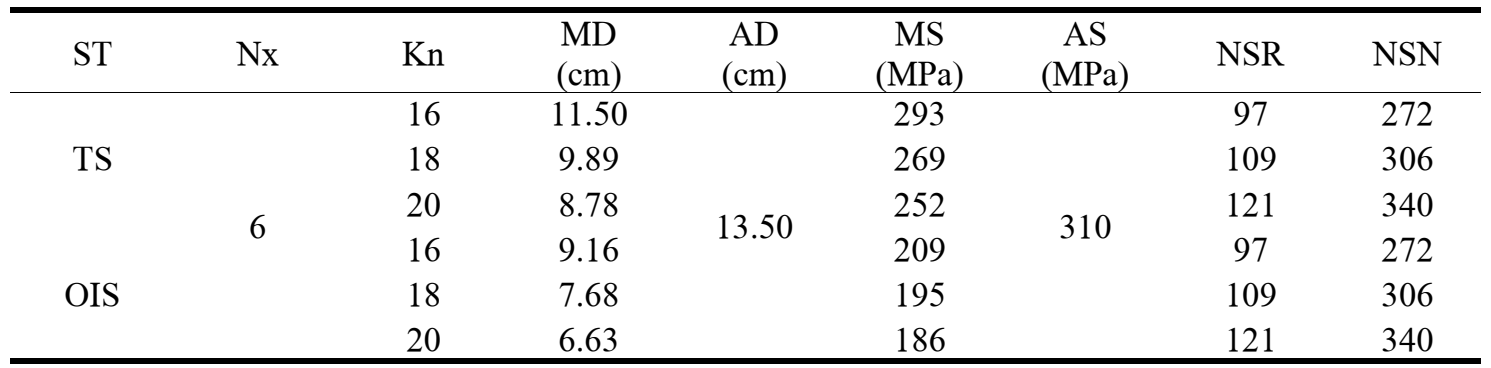

Table 3. $\mathrm{Nx}=8$, contrasting between $\mathrm{TF}$ and $\mathrm{OF}$ under different $\mathrm{Kn}$.

\begin{tabular}{|c|c|c|c|c|c|c|c|c|}
\hline ST & $\mathrm{Nx}$ & $\mathrm{Kn}$ & $\begin{array}{l}\mathrm{MD} \\
(\mathrm{cm})\end{array}$ & $\begin{array}{l}\mathrm{AD} \\
(\mathrm{cm})\end{array}$ & $\begin{array}{l}\mathrm{MS} \\
(\mathrm{MPa})\end{array}$ & AS (MPa) & NSR & NSN \\
\hline \multirow{4}{*}{$\mathrm{TS}$} & \multirow{6}{*}{8} & 16 & 14.32 & \multirow{6}{*}{13.50} & 347 & \multirow{6}{*}{310} & 129 & 368 \\
\hline & & 18 & 11.72 & & 295 & & 145 & 414 \\
\hline & & 20 & 9.99 & & 261 & & 161 & 460 \\
\hline & & 16 & 12.40 & & 267 & & 129 & 368 \\
\hline \multirow[t]{2}{*}{ OIS } & & 18 & 9.80 & & 218 & & 145 & 414 \\
\hline & & 20 & 8.07 & & 189 & & 161 & 460 \\
\hline
\end{tabular}

Note: the shadow data in the table has exceeded the allowable value of the structure. 
Table 4. $\mathrm{Nx}=10$, contrasting between $\mathrm{TF}$ and $\mathrm{OF}$ under different $\mathrm{Kn}$.

\begin{tabular}{|c|c|c|c|c|c|c|c|c|}
\hline ST & $\mathrm{Nx}$ & $\mathrm{Kn}$ & $\begin{array}{l}\mathrm{MD} \\
(\mathrm{cm})\end{array}$ & $\mathrm{AD}(\mathrm{cm})$ & $\begin{array}{l}\mathrm{MS} \\
(\mathrm{MPa})\end{array}$ & $\begin{array}{l}\mathrm{AS} \\
(\mathrm{MPa})\end{array}$ & NSR & NSN \\
\hline \multirow{4}{*}{ TS } & \multirow{6}{*}{10} & 16 & 18.67 & \multirow{6}{*}{13.50} & 451 & \multirow{6}{*}{310} & 161 & 464 \\
\hline & & 18 & 14.65 & & 369 & & 181 & 522 \\
\hline & & 20 & 12.05 & & 301 & & 201 & 580 \\
\hline & & 16 & 17.21 & & 371 & & 161 & 464 \\
\hline \multirow[t]{2}{*}{ OIS } & & 18 & 13.07 & & 294 & & 181 & 522 \\
\hline & & 20 & 10.38 & & 245 & & 201 & 580 \\
\hline
\end{tabular}

Note: the shadow data in the table has exceeded the allowable value of the structure. 4:

The following conclusions can be drawn from table 2-

(1). Two types of grid net shell in the process of Kn gradually increase, MS and MD gradually become smaller, and $\mathrm{Kn}$, the greater the smaller amplitude is smaller, but NSR and NSN have greatly increased, this means that to meet $\mathrm{AD}$ and $\mathrm{AS}$ conditions, can greatly save timber structure by reducing $\mathrm{Kn}$.

(2). Comparing the two grid types under the same Kn and Nx conditions, the MD and MS of the OIS are smaller than that of the TS, When Kn changes, the change value of the MS corresponding to the OIS is significantly smaller than that of the TS, indicating that the mechanical performance of the TS is sensitive to the change of $\mathrm{Kn}$.

(3). In the range of $\mathrm{Nx}=6-10$, under the same $\mathrm{Kn}$ condition, with the increase of Nx, the MD and MS of the two grid types increase. Under different $\mathrm{Nx}$ conditions, the OIS is still better than that of the TS.

\section{Conclusion}

(1). The reticulated shell with grid optimization method has better mechanical performance.

(2). Mesh optimization can not change the most unfavorable position, but can reduce the most unfavorable stress.

(3). The selection of appropriate Kn parameters can make the reticulated shell greatly save structural materials under the condition that the strength design requirements are met, and the mechanical performance of the mesh optimized reticulated shell is not sensitive to the change of $\mathrm{Kn}$. Therefore, the optimization of the isosceles triangular mesh is more conducive to the selection of smaller $\mathrm{Kn}$ parameters and the structure can save materials.

(4). The mesh shell optimized by the optimization model has more congruent triangles in its mesh, which greatly reduces the type of bars and brings great convenience to the engineering construction. Reasonable grid planning is of great significance to engineering practice.

\section{References}

1. Lu, X.Y., Zhao, X.W., Chen, S.Y. (2013) Discrete Design Variables Structural Optimization Design. China Architecture \& Building Press, BeiJing.

2. Tian, W., Dong, S.L., Zhao, Y. (2012) Planar quadrilateral meshing of spherical reticulated shell structure. Spatial Structures, 18: 43-48.

3. Liu, K.G., (1990) Practical calculation of regular triangle mesh sphere shell. Huazhong Architecture, 1990: 33-40.

4. Yang, A.D., Zhang, F.W., (1999) Discussion on the optimal method of triangular mesh to split orbital triangle. Spatial Structures, 5: 47-53.

5. Huang, Y.H., Xu, Q.X., (2018) On the ellipsoid and plane intersection equation. Journal of Shanghai Normal University (Natural Sciences), 47: 24-30.

6. Xin, J., Parametric Design and Stability Analysis of Single-layer Ellipsoidal Reticulated Shell, Master, Shandong Jianzhu University, JiNan, 2015.

7. Li, Z., Bi, P.B., Zhang, W.F., (2003) Static analysis of China National Grand Theater structure. Shanxi Architecture, 2003: 1-2.

8. Chen, Z.H., Liu, H.B., Zhou, T., (2013) Parameterized Calculation and Analysis of Space Steel Structure APDL. China Water \& Power Press, BeiJing.

9. Of the People's Republic of China national standard GB50009-2012 load code for the design of building structures.

10. Of the People's Republic of China national industry standard JGJ 7-2010 technical specification for space frame structures. 